\title{
Processos oxidativos avançados: fundamentos e aplicação ambiental
}

\author{
Mariele Fioreze', Eliane Pereira dos Santos ${ }^{2}$, Natana Schmachtenberg ${ }^{3}$
}

1,2,3 Universidade Federal de Santa Maria, Santa Maria, RS, Brasil

\section{Resumo}

Os Processos Oxidativos Avançados (ou Processos Avançados de Oxidação), conhecidos pela sigla POA's, são processos que se baseiam na geração de radicais livres, principalmente o radical hidroxil $(\cdot \mathrm{OH})$, que possui alto poder oxidante e pode promover a degradação de vários compostos poluentes eficientemente. Esses processos têm obtido grande atenção devido ao aumento da complexidade e dificuldade no tratamento de águas residuárias, o que tem sido motivo para a busca de novas metodologias visando a remediação desses rejeitos. Diante disso, o presente trabalho teve por objetivo relacionar quatro metodologias de tratamento via Oxidação Avançada: processos envolvendo H2O2, sistemas Fenton e Foto-Fenton, Fotocatálise Heterogênea e sistemas fundamentados na utilização de ozônio. A revisão realizada descreve os principais fundamentos destes processos e discute mecanismos de degradação de poluentes orgânicos. As principais vantagens da utilização destes processos estão em sua inespecificidade, podendo ser utilizados para degradar substratos de qualquer natureza química, na possibilidade de emprego para degradar poluentes cuja concentração seja muito baixa ( $\mu$ g L-1) e na não geração de resíduos. Por outro lado, algumas desvantagens são observadas, como os custos que podem ser elevados e a formação de subprodutos de reação.

Palavras-chave: Oxidação Avançada. Processos Químicos. Tratamento de Efluentes.

\begin{abstract}
The Advanced Oxidation Processes (or Advanced Processes Oxidation) known by the acronym POA's are processes which rely on the generation of free radicals, especially the hydroxyl radical $(\cdot \mathrm{OH})$, which has high oxidizing power and can promote the degradation of various compounds pollutants efficiently. These processes have gained great attention due to the increasing complexity and difficulty in treating wastewater, which has been the reason for searching for new methodologies aimed at remediation of such wastes. Therefore, the present study aimed to relate four treatment methodologies of Advanced Oxidation: $\mathrm{H} 2 \mathrm{O} 2$ processes involving, systems Fenton and Photo-Fenton, Heterogeneous Photocatalysis and systems based on the use of ozone. The present review describes the main principles of these processes and discusses mechanisms of degradation of organic pollutants. The main advantages of using these processes is in its specificity and can be used to degrade substrates of any chemical nature, the possibility of employment to degrade pollutants whose concentration is very low ( $\mu \mathrm{g}$ L-1) and not waste. Furthermore, some drawbacks are noted, such costs can be high and the formation of reaction byproducts.
\end{abstract}

Keywords: Advanced Oxidation. Chemical Processes. Wastewater Treatment. 


\section{INTRODUÇÃO}

O desenvolvimento tecnológico, consolidado a partir da Revolução Industrial (século XVIII), marcou o início de grandes evoluções nos processos industriais. Tais processos se tornaram essenciais para a sociedade moderna, a fim de atender às crescentes demandas de uma população em expansão. Visando sanar as necessidades básicas desta população em crescimento, o homem desenvolveu uma infinidade de técnicas para processar matérias-primas, modificando muito rapidamente a forma de exploração dos recursos naturais e intensificando a exploração do meio ambiente (SILVA, 2007).

Porém, essa evolução ficou associada a inúmeros problemas ambientais. Dois fatores aos quais pode ser associada à contaminação ambiental são: o acúmulo de matérias-primas e insumos, os quais envolvem uma série de riscos de contaminação por transporte e disposição inadequada, e ineficiência dos processos de conversão, o que necessariamente implica na geração de resíduos (FREIRE et al., 2000a).

A problemática causada pelos poluentes orgânicos industriais tem aumentado à necessidade de que as indústrias arquem com as responsabilidades do controle de seus efluentes. Dentre os diferentes processos para promover a adequação dos efluentes industriais, o tratamentos biológicos são atualmente os mais utilizados, devido aos baixos custos de implantação e operação e a possibilidade de tratar grandes volumes (KOSITZI et al., 2004). Porém, este tipo de tratamento possui limitações que podem restringir sua eficiência no tratamento de determinadas classes de efluentes com elevada toxicidade, como os organoclorados e fenóis (SILVA, 2007; FEITKENHAUER et al., 2003; SERPONE, 1997).

Os processos químicos vêm apresentando uma enorme aplicabilidade em sistemas de depuração ambiental. Porém, métodos como a precipitação e a incineração, apesar de amplamente utilizados, são bastante discutíveis, devido ao fato de o primeiro causar uma simples mudança de fase dos compostos sem eliminá-los, e do segundo possuir alto custo e possibilidade de formação de compostos mais tóxicos que o próprio efluente (FREIRE et al., 2000a).

Desta forma, é observada uma grande necessidade de desenvolver procedimentos que apresentem maior eficiência no tratamento de efluentes. Dentre as novas tecnologias, se destacam os Processos Oxidativos Avançados (POA's), os quais se baseiam na utilização de espécies altamente oxidantes para promover uma degradação mais efetiva do poluente a ser tratado (BRITO \& SILVA, 2012). Os POA's podem ser utilizados em conjunto com tratamentos biológicos, a fim de aumentar a biodegradabilidade de compostos recalcitrantes, diminuindo assim o tempo requerido para o tratamento via processos biológicos tradicionais (DE MORAES \& PERALTA-ZAMORA, 2005).

Neste trabalho, são abordadas quatro metodologias de tratamento via Oxidação Avançada envolvendo $\mathrm{H}_{2} \mathrm{O}_{2}$, sistemas Fenton e Foto-Fenton, Fotocatálise Heterogênea e sistemas fundamentados na utilização de ozônio, por meio da avaliação dos mecanismos envolvidos, eficiência da técnica, e suas vantagens e desvantagens.

\section{PROCESSOS OXIDATIVOS AVANÇADOS}

\section{I CONSIDERAÇÕES INICIAIS}

Os Processos Oxidativos Avançados (POA's) são baseados na geração de radicais livres, em especial o radical hidroxil $(\cdot \mathrm{OH})$. Este radical possui alto poder oxidante e pode promover a degradação de vários compostos poluentes em poucos minutos (HIRVONEN et al., 1996; NOGUEIRA \& JARDIM, 1998; SILVA, 2007; VINODGOPAL et al., 1998).

O radical hidroxil reage rápida e indiscriminadamente com muitos compostos orgânicos de diferentes formas, como por adição à dupla ligação ou por abstração do átomo de hidrogênio em moléculas orgânicas alifáticas (SOUZA et al., 2010; TIBURTIUS \& PERALTA-ZAMORA, 2004). O resultado dessas reações é a formação de radicais orgânicos que reagem com oxigênio, dando assim

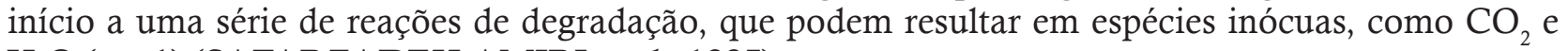
$\mathrm{H}_{2} \mathrm{O}$ (eq. 1) (SAFARZADEH-AMIRI et al., 1997).

$\cdot \mathrm{OH}+\mathrm{e}^{-}+\mathrm{H}^{+} \rightarrow \mathrm{H}_{2} \mathrm{O}$

(eq. 1)

O radical hidroxil é geralmente formado através de reações que resultam da combinação de oxidantes, como o ozônio $\left(\mathrm{O}_{3}\right)$ e o peróxido de hidrogênio $\left(\mathrm{H}_{2} \mathrm{O}_{2}\right)$, com radiação ultravioleta (UV) 
ou visível (Vis), e catalizadores, como íons metálicos ou semicondutores (NOGUEIRA et al., 2007).

Os POA's podem ser divididos em sistemas homogêneos e heterogêneos, conforme aparentando na Tabela 1 (SOUZA, 2010).

Tabela 1. Sistemas típicos de Processos Oxidativos Avançados.

\begin{tabular}{|c|c|c|}
\hline Processo & Homogêneo & Heterogêneo \\
\hline \multirow{4}{*}{ Com irradiação } & $\mathrm{O}_{3} / \mathrm{UV}$ & \multirow{4}{*}{$\begin{array}{l}\text { Fotocatálise Heterogênea } \\
\qquad\left(\mathrm{TiO}_{2} / \mathrm{O}_{2} / \mathrm{UV}\right.\end{array}$} \\
\hline & $\mathrm{H}_{2} \mathrm{O}_{2} / \mathrm{UV}$ & \\
\hline & $\mathrm{O}_{3} / \mathrm{H}_{2} \mathrm{O}_{2} / \mathrm{UV}$ & \\
\hline & Foto-Fenton & \\
\hline \multirow{3}{*}{ Sem irradiação } & $\mathrm{O}_{3} / \mathrm{HO}^{-}$ & \multirow{3}{*}{$\mathrm{O}_{3} /$ catalisador } \\
\hline & $\mathrm{O}_{3} / \mathrm{H}_{2} \mathrm{O}_{2}$ & \\
\hline & Reativo de Fenton & \\
\hline
\end{tabular}

Dentre as principais vantagens associadas ao uso de tecnologias fundamentadas em Processos Oxidativos Avançados, podem ser citadas:

- Os POA's introduzem importantes modificações químicas no substrato, podendo inclusive levar a sua completa mineralização (SARRIA et al., 2002; TABRIZI \& MEHRVAR, 2004);

- A inespecificidade dos POA's viabiliza a sua utilização para a degradação de substratos de qualquer natureza química, sendo destaque a degradação de contaminantes refratários e tóxicos, cujo tratamento biológico pode ser viabilizado através da oxidação avançada parcial (GOGATE \& PANDIT, 2004; KIWI et al., 1994; PARRA, 2001; SCOTT \& OLLIS, 1995);

- Os POA's podem ser aplicados para reduzir a concentração de compostos formados em etapas de pré-tratamento, como por exemplo, aromáticos halogenados formados durante a desinfecção convencional (TABRIZI \& MEHRVAR, 2004);

- Os POA's podem ser aplicados no tratamento de contaminantes cuja concentração seja muito baixa (ppb, por exemplo) (DOMÈNECH et al., 2001; GOGATE \& PANDIT, 2004);

- Na maioria dos casos, os POA's não geram resíduos, com exceção de alguns processos que podem envolver precipitação (por exemplo, óxidos férricos) (MORAIS, 2005).

- Assim como qualquer outro tratamento, a Oxidação Avançada não pode ser aplicada indiscriminadamente a qualquer tipo de resíduo (MORAIS, 2005). Dentre as condições que limitam sua aplicabilidade, se destacam (DOMÈNECH et al., 2001; FREIRE et al., 2000b):

- Nem todos os processos estão disponíveis em escala adequada;

- Os custos podem ser elevados, principalmente devido ao consumo de energia;

- Há formação de subprodutos de reação, os quais em alguns casos são tóxicos;

- Apresentam restrições de aplicação em condições de elevada concentração dos poluentes;

- Dentre os processos de geração do radical hidroxil, se destacam os sistemas que utilizam ozônio, peróxido de hidrogênio, a Fotocatálise Heterogênea e Reagente de Fenton (FREIRE et al., 2000a).

- Os contaminantes orgânicos podem ser oxidados pelo radical hidroxil de acordo com diferentes mecanismos básicos. Estes mecanismos dependem da estrutura do contaminante orgânico, sendo estes: a) abstração de átomo de hidrogênio; b) transferência eletrônica; e c) adição eletrofílica (BRITO \& SILVA, 2012; NOGUEIRA et al., 2007). 


\section{I.I Abstração de átomo de hidrogênio}

Os radicais orgânicos são formados a partir da oxidação de compostos orgânicos por abstração de hidrogênio, causada pelos radicais hidroxil (eq. 2) (NOGUEIRA et al., 2007; MARTINS, 2011). Posteriormente, o radical peróxido é formado através da adição de oxigênio molecular (eq. 3), intermediário que inicia reações térmicas em cadeia levando à degradação à $\mathrm{CO}_{2}$, água e sais orgânicos (BRITO \& SILVA, 2012). Este tipo de reação ocorre geralmente com hidrocarbonetos alifáticos.

$\cdot \mathrm{OH}+\mathrm{RH} \rightarrow \mathrm{R} \cdot+\mathrm{H}_{2} \mathrm{O}$

(eq. 2)

$\mathrm{R} \cdot+\mathrm{O}_{2} \rightarrow \mathrm{RO}_{2}$

(eq. 3)

\subsubsection{Adição eletrofílica}

Ocorre em compostos orgânicos que contêm ligações $\pi$, sendo que o resultado dessas reações são os radicais orgânicos (eq. 4) (NOGUEIRA et al., 2007; MARTINS, 2011). Essas reações ocorrem geralmente com hidrocarbonetos insaturados ou aromáticos.<smiles>[R]C([R])=C([R])[Hg]OCC</smiles>

(eq. 4)

\section{I.3 Transferência eletrônica}

As reações de transferência eletrônica ocorrem em casos onde a adição eletrofílica e a abstração de hidrogênio são desfavorecidas, como acontece com hidrocarbonetos clorados (eq. 5) (MARTINS, 2011; NOGUEIRA et al., 2007).

$$
\mathrm{RX}+\cdot \mathrm{OH} \rightarrow \mathrm{RX}^{+}+\mathrm{OH}^{-}
$$

(eq. 5)

\subsection{Processos Oxidativos Avançados envolvendo $\mathrm{H}_{2} \mathrm{O}_{2}$ (sistema UV/ $\mathrm{H}_{2} \mathrm{O}_{2}$ )}

$O$ radical hidroxil pode ser eficientemente gerado a partir de sistemas que envolvem peróxido de hidrogênio $\left(\mathrm{H}_{2} \mathrm{O}_{2}\right)$ e radiação ultravioleta (UV) (eq. 6) (TIBURTIUS \& PERALTA-ZAMORA, 2004). A produção do radical hidroxil envolve a cisão homolítica da molécula de peróxido, o que ocorre com a quebra de uma ligação sigma de elevada energia (MORAIS, 2005).

$\mathrm{H}_{2} \mathrm{O}_{2}+h v(254 \mathrm{~nm}) \rightarrow 2 \cdot 0 \mathrm{H}$

(eq. 6)

A formação do radical HO ocorre devido à oxidação direta peróxido através da irradiação UV (eq. 7, 8 e 9) (SOUZA et al., 2010).

$$
\begin{aligned}
& \mathrm{H}_{2} \mathrm{O}_{2}+h v \rightarrow 2 \mathrm{HO} \cdot \\
& \text { (eq. 7) } \\
& \mathrm{H}_{2} \mathrm{O}_{2}+\mathrm{O}_{2}^{-} \rightarrow \mathrm{HO} \cdot+\mathrm{HO}^{-}+\mathrm{O}_{2} \\
& \text { (eq. 8) } \\
& \begin{array}{l}
\mathrm{RH}+\mathrm{HO} \cdot \rightarrow \mathrm{H}_{2} \mathrm{O}+\mathrm{R} \cdot \rightarrow \text { produtos oxidados } \\
\text { (eq. 9) }
\end{array}
\end{aligned}
$$


O emprego do peróxido de hidrogênio combinado com radiação ultravioleta resulta numa reação indireta, muito mais eficiente devido ao fato que o potencial de oxidação do radical hidroxil $\left(\mathrm{E}^{0}=+2,80 \mathrm{~V}\right)$ é mais elevado que o do peróxido de hidrogênio molecular $\left(\mathrm{E}^{0}=+1,78 \mathrm{~V}\right)$, podendo assim, promover oxidação mais enérgica (BRITO \& SILVA, 2012).

O peróxido de hidrogênio também pode agir como capturador de radicais HO· (eq. 10) (SOUZA et al., 2010), comprometendo assim o sistema.

$\mathrm{H}_{2} \mathrm{O}_{2}+\mathrm{HO} \cdot \rightarrow \mathrm{H}_{2} \mathrm{O}+\mathrm{HO}_{2}^{-}$

(eq. 10)

A Tabela 2 apresenta as vantagens e as desvantagens do uso do sistema $\mathrm{UV} / \mathrm{H}_{2} \mathrm{O}_{2}$.

Tabela 2. Vantagens e desvantagens do processo $\mathrm{UV} / \mathrm{H}_{2} \mathrm{O}_{2}$.

\begin{tabular}{cc}
\hline Vantagens & Desvantagens \\
\hline Solubilidade do $\mathrm{H}_{2} \mathrm{O}_{2}$ em água & Custo do processo \\
$\begin{array}{c}\text { Geração de dois radicais } \mathrm{HO} \text { p por } \\
\text { molécula de } \mathrm{H}_{2} \mathrm{O}_{2} \text { fotolisada }\end{array}$ & $\mathrm{H}_{2} \mathrm{O}_{2}$ funciona como um “capturador" \\
Estabilidade térmica & de radicais hidroxil \\
Procedimentos de operações simples & $\begin{array}{c}\text { Taxa de oxidação química do poluente } \\
\text { é limitada pela taxa de formação dos } \\
\text { Inexistência de problemas de } \\
\text { transferência de massa }\end{array}$ \\
\end{tabular}

Vários poluentes orgânicos presentes em efluentes industriais tem sido efetivamente decompostos através da geração de radicais hidroxil utilizando $\mathrm{H}_{2} \mathrm{O}_{2}$ e $h v$ (INCE et al., 2002; SCHRANK et al., 2005)

\subsection{Tratamento via Reação de Fenton e Foto-Fenton (sistemas $\mathrm{Fe}^{2+} / \mathrm{H}_{2} \mathrm{O}_{2}$ e Fe $\mathrm{Fe}^{2+} / \mathrm{H}_{2} \mathrm{O}_{2} / \mathrm{UV}$ ou Vis)}

A Reação de Fenton consiste na reação redox de decomposição do $\mathrm{H}_{2} \mathrm{O}_{2}$ catalisada por $\mathrm{Fe}^{3+}$ (eq. 11), que leva à geração de um radical hidroxil para cada mol de peróxido de hidrogênio presente no meio (BRITO \& SILVA, 2012).

$$
\begin{aligned}
& \mathrm{H}_{2} \mathrm{O}_{2}+\mathrm{Fe}^{2+} \rightarrow \mathrm{OH}^{-}+\cdot \mathrm{OH}+\mathrm{Fe}^{3+} \\
& \text { (eq. 11) }
\end{aligned}
$$

Os íons ferrosos $\left(\mathrm{Fe}^{2+}\right)$ agem como catalizadores para a decomposição do peróxido de hidrogênio em meio ácido, gerando o radical hidroxil (SOUZA et al., 2010). A oxidação a partir do processo Fenton pode ocorrer de maneira direta, onde íons $\mathrm{Fe}^{2+} \mathrm{e} \mathrm{Fe}^{3+}$ podem reduzir ou oxidar diretamente a matéria orgânica (eq. 12), e indireta, onde há a formação do radical hidroxil (eq. 13) (BRITO \& SILVA, 2012).

$$
\begin{aligned}
& \mathrm{Fe}^{2+}+\mathrm{Cl}-\mathrm{RH} \rightarrow \mathrm{Fe}^{3+}+\mathrm{Cl}^{-}+\mathrm{RH} \\
& \mathrm{Fe}^{3+}+\mathrm{R} \rightarrow \mathrm{Fe}^{2+}+\mathrm{R}^{+} \\
& \text {(eq. 12) }
\end{aligned}
$$


$\cdot \mathrm{OH}+\mathrm{R} \rightarrow \mathrm{Fe}^{2+}+\mathrm{R}^{+}$

(eq. 13)

Porém, o $\mathrm{H}_{2} \mathrm{O}_{2}$ pode também atuar como sequestrados do radical hidroxil, formando o radical hidroperoxil $\left(\mathrm{HO}_{2} \cdot\right)$, que possui menor potencial de redução $\left(\mathrm{E}^{0}=1,42 \mathrm{~V}\right)$ que o radical hidroxil, prejudicando assim o processo de degradação (eq. 14) (NOGUEIRA et al., 2007).

$\mathrm{H}_{2} \mathrm{O}_{2}+\cdot \mathrm{OH} \rightarrow \mathrm{HO}_{2} \cdot+\mathrm{H}_{2} \mathrm{O}$

(eq. 14)

A potencialidade do uso desse processo para o tratamento de efluentes se deve principalmente à sua simplicidade, uma vez que a reação ocorre à temperatura e pressão ambientes e se aplica a uma grande variabilidade de compostos, além de o ferro ser o quarto elemento mais abundante da crosta terrestre (BRITO \& SILVA, 2012; NOGUEIRA et al., 2007).

Nos anos 80, foi descoberto que a irradiação do Reagente de Fenton provocava a fotorredução dos íons $\mathrm{Fe}^{3+}$ previamente formados, com geração de mais de um mol do íon hidroxil (eq. 15) (BRITO \& SILVA, 2012). Essa reação, conhecida como Reação de Foto-Fenton, é menos favorecida que a Reação de Fenton, com uma constante $\mathrm{k}=0,02 \mathrm{~L} \cdot \mathrm{mol}^{-1} \cdot \mathrm{s}^{-1}$ (a reação de Fenton possui $\mathrm{k}=76 \mathrm{~L} \cdot \mathrm{mol}^{-1} \cdot \mathrm{s}^{-1}$ a $30^{\circ} \mathrm{C}$ e $\left.\mathrm{pH}=3,0\right)$ (BRITO \& SILVA, 2012; NOGUEIRA et al., 2007). Na prática, isso significa que, embora as duas reações ocorram concomitantemente em um meio racional, há a predominância de íons férricos, o que determina que o $\mathrm{pH}$ deve ser mantido abaixo de 4,0 para evitar a precipitação de oxi-hidróxidos férricos (BRITO \& SILVA, 2012).

$\mathrm{Fe}^{3+}+\mathrm{H}_{2} \mathrm{O}_{2}+h v\left(\mathrm{UV}\right.$ ou Vis) $\rightarrow \mathrm{Fe}^{2+}+\mathrm{H}^{+}+\cdot \mathrm{OH}$

(eq. 15)

Uma vez que os processos Fenton e Foto-Fenton são catalisados por íons $\mathrm{Fe}^{2+} / \mathrm{Fe}^{3+}$ e considerando que estes íons são hidrolisados formando hidróxidos insolúveis, o $\mathrm{pH}$ do meio tem papel importante nas reações envolvidas, o que consequentemente afeta a velocidade de degradação do composto orgânico (NOGUEIRA et al., 2007). É observado que uma pequena faixa de $\mathrm{pH}$, entre 2,5 e 3,0, proporciona a máxima eficiência de degradação (NOGUEIRA \& GUIMARÃES, 2000; PIGNATELLO, 1992). Esta estreita faixa de $\mathrm{pH}$ é decorrência da precipitação de $\mathrm{Fe}(\mathrm{III})$ em valores de $\mathrm{pH}$ acima de 3,0 , diminuindo drasticamente sua interação com o peróxido de hidrogênio e, consequentemente, a produção de $\cdot \mathrm{OH}$ (NOGUEIRA et al., 2007). Abaixo do $\mathrm{pH}$ 2,5, a velocidade de degradação também é afetada, apesar das espécies de Fe permanecerem solúveis, pois as altas concentrações de $\mathrm{H}^{+}$podem sequestrar radicais hidroxil (eq. 16) (SPINKS \& WOODS, 1990).

$$
\cdot \mathrm{OH}+\mathrm{H}^{+}+\mathrm{e}^{-} \rightarrow \mathrm{H}_{2} \mathrm{O}
$$

(eq. 16)

Complexos orgânicos de ferro também têm sido empregados na degradação de contaminantes em reações Foto-Fenton, devido a estabilização do ferro em uma faixa mais ampla de pH em relação àquela na ausência de complexos, além de os complexos de ferro contribuírem para o aumento da eficiência de absorção da luz, uma vez que estes estendem a banda de absorção para a região do visível (NOGUEIRA et al., 2007; ZUO \& HOIGNÉ, 1992).

O ferrioxalato de potássio (FeOx) é um complexo de $\mathrm{Fe}(\mathrm{III})$ bastante estudado, tanto em aplicações fotoquímicas como em processos Foto-Fenton (NOGUEIRA et al., 2007). A fotólise deste complexo é bastante sensível à irradiação em comprimentos de onda entre 200 e $500 \mathrm{~nm}$, gerando $\mathrm{Fe}^{2+}$ e $\mathrm{CO}_{2}$ em meio ácido (eq. 17, 18 e 19) (NOGUEIRA et al., 2007).

$$
\begin{aligned}
& {\left[\mathrm{Fe}\left(\mathrm{C}_{2} \mathrm{O}_{4}\right)_{3}\right]^{3-}+h v \rightarrow\left[\mathrm{Fe}\left(\mathrm{C}_{2} \mathrm{O}_{4}\right)_{2}\right]^{2-}+\mathrm{C}_{2} \mathrm{O}_{4}^{--}} \\
& (\text {eq. 17) } \\
& \mathrm{C}_{2} \mathrm{O}_{4}^{-\cdot}+\left[\mathrm{Fe}\left(\mathrm{C}_{2} \mathrm{O}_{4}\right)_{3}\right]^{3-} \rightarrow\left[\mathrm{Fe}\left(\mathrm{C}_{2} \mathrm{O}_{4}\right)_{2}\right]^{2-}+\mathrm{C}_{2} \mathrm{O}_{4}{ }^{2-}+2 \mathrm{CO}_{2} \\
& (\text { eq. 18) }
\end{aligned}
$$


$\mathrm{C}_{2} \mathrm{O}_{4}^{-\cdot}+\mathrm{O}_{2} \rightarrow \mathrm{O}_{2}^{-\cdot}+2 \mathrm{CO}_{2}$

(eq. 19)

\subsection{Fotocatálise Heterogênea (sistema TiO $/ 2$ UV)}

Os processos de Fotocatálise Heterogênea tiveram origem nos anos 70, quando pesquisas em células fotoeletroquímicas começaram a ser desenvolvidas com o intuito de produzir combustíveis a partir de materiais baratos, visando à transformação da energia solar em química (BRITO \& SILVA, 2012).

A aplicação da fotocatálise visando à descontaminação foi explorada pela primeira vez em dois trabalhos de Pruden e Ollis (PRUDEN \& OLLIS, 1983a; PRUDEN \& OLLIS, 1983b), onde foi estudada a degradação de clorofórmio e tricloroetileno para íons orgânicos durante iluminação de suspenção de $\mathrm{TiO}_{2}$ (BRITO \& SILVA, 2012).

A Fotocatálise Heterogênea consiste num processo onde uma espécie semicondutora é irradiada para a promoção de um elétron da banda de valência (BV) para a banda de condução (BC) (FREIRE et al., 2000a; TIBURTIUS \& PERALTA-ZAMORA, 2004; ZIOLLI \& JARDIM, 1998). Com o elétron promovido para a BC e com a lacuna $\left(h^{+}\right)$gerada na BV, são criados sítios redutores e oxidantes, que são capazes de catalisar reações químicas (eq. 20) (Figura 1) (SILVA, 2007; FREIRE et al., 2000a; ZIOLLI \& JARDIM, 1998). Os potenciais adquiridos são suficientes para gerar radicais $\cdot$ OH a partir de moléculas de água absorvidas na superfície do semicondutor (eq. 21) (ZIOLLI \& JARDIM, 1998), os quais podem subsequentemente oxidar o contaminante orgânico.

$$
\begin{aligned}
& \mathrm{TiO}_{2}+h v \rightarrow \mathrm{TiO}_{2}\left(\mathrm{e}_{\mathrm{BC}}^{-}+\mathrm{h}^{+}{ }_{\mathrm{BV}}\right) \\
& (\text { eq. 20) } \\
& \mathrm{h}^{+}+\mathrm{H}_{2} \mathrm{O}_{(\text {ads })} \rightarrow \cdot \mathrm{OH}+\mathrm{H}^{+} \\
& \text {(eq. 21) }
\end{aligned}
$$

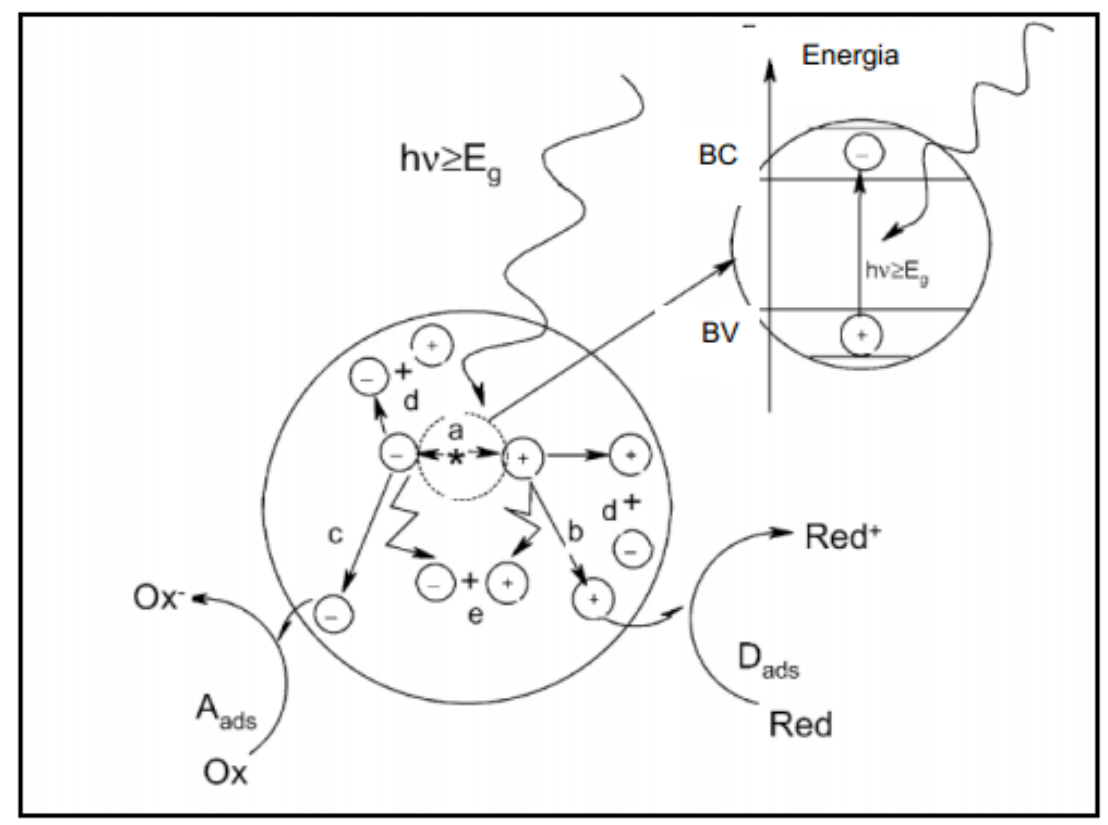

Figura 1. Principais processos ocorridos na partícula do semicondutor quando fotoexcitado: a) geração do par $\mathrm{e}_{\mathrm{BC}}^{-} / \mathrm{h}^{+}{ }_{\mathrm{BV}}$; b) oxidação do doador (D); c) redução do aceptor (A); d) recombinação do par $\mathrm{e}_{\mathrm{BC}}^{-} / \mathrm{h}^{+}{ }_{\mathrm{BV}}$ fotogerados na superfície e no cerne da partícula, respectivamente.

Na fotocatálise, a luz ultravioleta, com $\square<380 \mathrm{~nm}$, e, portanto, com energia superior à barreira entre a banda de valência e a banda de redução do $\mathrm{TiO}_{2}$, induz a formação de elétrons na banda 
de valência e de vacâncias positivas na banda de condução (VIANNA et al., 2008). Estas espécies carregadas podem se recombinar liberando a energia absorvida como calor, ou podem migrar para a superfície da partícula do fotocatalisador (VIANNA et al., 2008). Quando na presença de água ou do íon hidróxido, o radical hidroxil é formado na superfície do semicondutor.

A eficiência do processo de Fotocatálise Heterogênea depende da competição entre o processo em que o elétron é retirado da superfície do semicondutor pelo oxigênio, evitando o processo de recombinação do par elétron-lacuna (VANDEVIVERE et al., 1998).

O processo de oxidação pode ocorrer por via direta e indireta. O processo por via direta se dá quando a lacuna fotogerada na banda de valência do semicondutor reage diretamente com o composto orgânico (eq. 22) (HOFFMANN et al., 1995). Já o processo de oxidação por via indireta ocorre quando a lacuna fotogerada na banda de valência reage com a molécula de $\mathrm{H}_{2} \mathrm{O}$ adsorvida na superfície do semicondutor produzindo o radical hidroxil, o qual vai oxidar a matéria orgânica (eq. 23) (CHOI \& HOFFMANN, 1997; HOFFMANN et al., 1995; LINSEBIGLER et al., 1995).

$$
\begin{aligned}
& \mathrm{R}_{1(\text { ads })}+\mathrm{h}^{+}{ }_{\mathrm{BV}} \rightarrow \mathrm{R}_{1(\mathrm{ads})}+ \\
& \text { (eq. 22) } \\
& \text { OH }+\mathrm{R}_{1} \rightarrow \mathrm{R}_{2} \\
& \text { (eq. 23) }
\end{aligned}
$$

Um dos semicondutores mais utilizados em processos fotocatalíticos é o dióxido de titânio $\left(\mathrm{TiO}_{2}\right)$ (D'OLIVEIRA et al., 1991; HOFFMANN et al., 1995). Dentre as características favoráveis à utilização do $\mathrm{TiO}_{2}$ se destacam: a possibilidade de ativação através da luz solar, a insolubilidade em água, a estabilidade química numa ampla faixa de $\mathrm{pH}$, a possibilidade de imobilização em sólidos, o baixo custo e a ausência de toxicidade (FREIRE et al., 2000a). Outros semicondutores, como CdS, ZnO, $\mathrm{WO}_{3}, \mathrm{ZnS}$ e $\mathrm{Fe}_{2} \mathrm{SO}_{3}$, podem agir como sensibilizadores de processos de oxidação e redução mediados pela luz (WANG et al., 1998; YEBER et al., 1999).

A forma anátase é a estrutura do $\mathrm{TiO}_{2}$ que apresenta a fotoatividade, porém estudos comprovam que a estrutura rutílica também pode apresentar uma pequena fotoatividade (BRITO \& SILVA, 2012; ZIOLLI \& JARDIM, 1998). Estudos vêm sendo desenvolvidos com uma porcentagem de $75 \%$ anátase e $25 \%$ rutilo apresentando maior fotoatividade (BRITO \& SILVA, 2012; ZIOLLI \& JARDIM, 1998).

Apesar de existir muitos estudos práticos envolvendo o $\mathrm{TiO}_{2}$, seu mecanismo ainda não é completamente compreendido (FREIRE et al., 2000a). Porém, a maioria dos pesquisadores concorda com alguns passos, tais como: a excitação da espécie condutora e a consequente formação dos pares $\mathrm{h}^{+}{ }_{\mathrm{BV}} \mathrm{e}$

$\mathrm{e}_{\mathrm{BC}}^{-}$, o processo de recombinação entre elas, a adsorção de $\mathrm{O}_{2}, \mathrm{H}_{2} \mathrm{O}$ e espécies orgânicas na superfície do semicondutor, "trapping" em que espécies químicas doam ou recebem elétrons do par $\mathrm{e}^{-} / \mathrm{h}^{+}$impedindo a recombinação. Ainda, supõe-se que o $\mathrm{O}_{2}$ é a espécie principal responsável em dar continuidade às reações iniciadas no processo de foto-oxidação, reagindo com o radical orgânico formado e o levando à completa mineralização (CHEN et al., 1999; CHOI \& HOFFMANN, 1997).

Quando utilizado em tratamento de efluentes contendo compostos organoclorados, o processo fotocatalítico tem demostrado eficiência (FREIRE et al., 2000a). Porém, a implementação destes processos em escala industrial apresenta alguns problemas, como por exemplo, o uso da luz ultravioleta que encarece o tratamento, visto que a construção de estações que utilizem luz solar continua sendo um desafio (NOGUEIRA \& JARDIM, 1998; PREVOT \& PRAMAURO, 1999), a imobilização do semicondutor gera perdas na atividade fotocatalítica (PERALTA-ZAMORA et al., 1997), e há a necessidade de separação das finas partículas do catalizador (KAGAYA et al., 1999).

\subsection{Sistemas Fundamentados em Ozônio $\left(\mathrm{O}_{3}\right)$}

$\mathrm{O}$ ozônio $\left(\mathrm{O}_{3}\right)$ é um oxidante energético e, por causa disto, é muito utilizado em processos de degradação de compostos orgânicos. O ozônio pode reagir através de mecanismo direto (reação eletrofílica ou por cicloadição), ou mecanismo indireto (através do radical livre hidroxil formado a partir da decomposição do ozônio) (eq. 24) (FREIRE et al., 2000b; MASTEN \& DAVIS, 1994). 
$\mathrm{O}_{3}+\mathrm{OH}^{-} \rightarrow \mathrm{O}_{2}^{-}+\mathrm{HO}_{2}$

$\mathrm{O}_{3}+\mathrm{HO}_{2} \cdot \rightarrow 2 \mathrm{O}_{2}+\cdot \mathrm{OH}$

(eq. 24)

Por meio da reação direta, o ozônio pode reagir com outras moléculas orgânicas via adição eletrofílica (MORAIS, 2005). O ataque eletrófilo do ozônio pode acontecer em átomos com uma densidade de carga negativa (N, $\mathrm{P}, \mathrm{O}$ ou carbonos nucleofílicos), ou a ligações duplas ou triplas do tipo carbono-carbono e nitrogênio-nitrogênio (eq. 25, 26, 27 e 28) (KUNZ, 1999).

$\mathrm{O}_{3}+\mathrm{NO}_{2}^{-} \rightarrow \mathrm{NO}_{3}^{-}+\mathrm{O}_{2}$

(eq. 25)

$\mathrm{O}_{3}+\mathrm{CN}^{-} \rightarrow \mathrm{CNO}^{-}+\mathrm{O}_{2}$

(eq. 26)

$\mathrm{O}_{3}+\mathrm{SO}_{3}{ }^{2-} \rightarrow \mathrm{SO}_{4}{ }^{2-}+\mathrm{O}_{2}$

(eq. 27)

$\mathrm{O}_{3}+\mathrm{R}_{2} \mathrm{C}=\mathrm{CR}_{2} \rightarrow \mathrm{RCHO}$

(eq. 28)

Por via indireta, o ozônio pode reagir através da formação de espécies radicalares (eq. 29, 30, 31 e 32), sendo o principal radical formado o hidroxil, o qual apresenta a capacidade de reagir com os compostos orgânicos $10^{6}$ a $10^{12}$ vezes mais rápido que o ozônio (MORAIS, 2005).

$\mathrm{O}_{3}+\mathrm{H}_{2} \mathrm{O}+h v \rightarrow \mathrm{H}_{2} \mathrm{O}_{2}+\mathrm{O}_{2}$

(eq. 29)

$\mathrm{H}_{2} \mathrm{O}_{2}+h v \rightarrow 2 \mathrm{HO}$.

(eq. 30)

$\mathrm{O}_{3}+\mathrm{HO}^{-} \rightarrow \mathrm{O}_{2}^{-}+\mathrm{HO}_{2}$.

(eq. 31)

$\mathrm{O}_{3}+\mathrm{HO}_{2} \cdot \rightarrow 2 \mathrm{O}_{2}+\mathrm{HO}$

(eq. 32)

Dentre os dois mecanismos de reação, a reação indireta é muito mais eficiente porque o potencial de oxidação do radical hidroxil $\left(\mathrm{E}^{0}=+3,06 \mathrm{~V}\right)$ é mais elevado que o do ozônio molecular $\left(\mathrm{E}^{0}=+2,07\right.$ $\mathrm{V}$ ), podendo assim promover uma oxidação mais enérgica (FREIRE et al., 2000a). Ainda, as reações com ozônio molecular tendem a ser mais seletivas (ataques a centros nucleofílicos), enquanto que os radicais hidroxil não reagem seletivamente (STOCKINGER et al., 1995), o que torna as reações por via indireta muito mais versáteis (FREIRE et al., 2000a). (eq. 33).

O ozônio também é capaz de gerar o radical hidroxil na presença de radiação ultravioleta $(h v)$

$\mathrm{O}_{3}+\mathrm{H}_{2} \mathrm{O}+h v \rightarrow \mathrm{H}_{2} \mathrm{O}_{2}+\mathrm{O}_{2}$

$\mathrm{H}_{2} \mathrm{O}_{2}+h v \rightarrow 2 \cdot \mathrm{OH}$

(eq. 33)

Também é possível gerar o radical hidroxil a partir de misturas de ozônio e hidróxido de hidrogênio, na ausência $\left(\mathrm{O}_{3} / \mathrm{H}_{2} \mathrm{O}_{2}\right)$ ou presença de radiação ultravioleta $\left(\mathrm{O}_{3} / \mathrm{H}_{2} \mathrm{O}_{2} / h v\right)$, ou simplesmente utilizando um meio fortemente alcalino $\left(\mathrm{O}_{3} / \mathrm{pH}\right.$ elevado) (FREIRE et al., 2000a). 


\section{CONCLUSÕES}

Os Processos Oxidativos Avançados são um conjunto de tecnologias promissoras para o tratamento de contaminantes persistentes e tóxicos, tendo grande aplicação para o tratamento de águas residuárias, devido à grande eficiência na rápida mineralização de espécies químicas de importância ambiental.

Porém, a aplicação destas tecnologias ainda demanda de mais estudos, uma vez que desenvolver e aperfeiçoar reatores em escala industrial ainda representa um dos grandes problemas para a implantação destes processos.

\section{REFERÊNCIAS}

BRITO, N. N. De.; SILVA, V. B. M. Processos Oxidativos Avançados e sua aplicação ambiental. Revista Eletrônica de Engenharia Civil, n.3, v.1, p.36-47, 2012.

CHEN, J.; OLLIS, D. F; RULKENS, W. H; BRUNING, H. Kinetic Processes of Photocatalytic Mineralization of Alco hols on Metallized Titanium Dioxide. Water Res. v.33, 1173, 1999.

CHOI, W. Y.; HOFFMANN, M. R. Novel photocatalytic mechanisms for $\mathrm{CHCl}_{3}, \mathrm{CHBr}_{3}$, and $\mathrm{CCl} 33 \mathrm{CO} 2-$ degradation and the fate of photogenerated trihalomethyl radicals on $\mathrm{TiO}_{2}$., Environ. Sci. Technol., v.31, p.89-95, 1997.

DE MORAIS, J. L.; PERALTA $\square$ ZAMORA, P. Use of advanced oxidation processes to improve the biodegradability of mature landfill leachates. Journal of Hazardous Materials, v.123, n.1 $\square$ 3, p 181 $\square$ 186, 2005.

D’OliveirA, J.; AL-SAYYeD, G.; PICHAT, P.; J. Photochem. Photobiol. A: Chem. 58, 99, 1991.

DOMÈNECH, X., JARDIM, W. F., LITTER, M. I. Procesos avanzados de oxidación para la eliminación de contaminantes. In: Eliminiación de Contaminantes por Fotocatálisis Heterogênea. Cap. 1, Rede CYTED, La Plata, 2001.

FEITKENHAUER, H.; SCHNICKE, S.; MÜLLER, R.; MÄRKL, H. Kinetic parameters of continuous cultures of Bacillus thermoleovorans sp. A2 degrading phenol at 65ï ¿ 1/2C. J. Biotechnol. 103, p.129-135, 2003.

FREIRE, R. S.; PELEGRINI, R.; KUBOTA, L. T.; DURÁN, N.; PERALTA-ZAMORA, P. Novas técnicas para o tratamento de resíduos industriais contendo espécies organocloradas. Química Nova, v.23, p.504-511, 2000a.

FREIRE, R. S.; KUNZ, A.; DURÁN, N. Some Chemical and Toxicological Aspects about Paper Mill Effluent Treatment with Ozone. Environ. Technol. v. 21, 717, 2000 b.

GOGATE, P. R.; PANDIT, A. B. A reviewof imperative technologies for wastewater treatment I. Oxidation technologies at amb conditions. Advanced Environmental Research. v.8, (3-4), p.501-551, 2004.

HIRVONEN, A.; TUHKANEN, T.; KALLIOKOSKI, P. Treatment of TCE- and PCE contaminated groundwater using UV/H2O2 and O3/H2O2 oxidation processes. Wat. Sci. Tech., v.33, p.67-73, 1996.

HOFFMANN, M. R.; MARTIN, S. T.; CHOI, W.; BAHNEMANN, D. W. Environmental Applications of Semiconductor Photocatalysis. Chem. Rev., v.95, p.69-96, 1995. 
INCE, N. H.; HASAN, D. A.; USTUN, B.; TEZCANLI, G. Combinative dyebath treatment with activated carbon and UV/H2O2: a case study on Everzol Black $\square$ GSP (R). Water Science Technology, v.46, n. $4 \square 5$, p. $51 \square 58,2002$.

KAGAYA, S; SHIMIZU, K.; ARAI, R.; HASEGAWA, K. Separation of Titanium Dioxide Photocatalyst in Its Aqueous Suspensions by Coagulation with Basic Aluminium Chloride. Water Res., v.33(7), p.1753-1755, 1999.

KIWI, J.; PULGRAIN, C.; PERINGER, P. Effect of Fenton and photo-Fenton reactions on the degradation and biodegradability of 2 and 4-nitrophenols in water treatment. Applied Catalysis. B: Environmental. v.3, p.335-341, 1994.

KOSITZI, M.; POULIOS, I.; MALATO, S.; CÁCERES, J.; CAMPOS, A. Solar photocatalytic treatment of synthetic municipal wastewater. Water Res, v.38 (5), p.1147-54, 2004.

KUNZ, A. Remediação de efluente têxtil: combinação entre processo químico (ozônio) e biológico. Tese (Doutorado em Química), UNICAMP, 1999.

LINSEBIGLER, A. L.; LU, G.; YATES Jr., J. T. Photocatalysis on TiOn Surfaces: Principles, Mechanisms, and Selected Results. Chem. Rev., v.95, p.735-758, 1995.

MASTEN, S. J.; DAVIS, S. H. R. The use of ozonation to degrade organic contaminants in wastewaters. Environ. Sci. Technol., v.28:180A-185A, 1994.

MARTINS, L. M. Estudo da aplicação de processos oxidativos avançados no tratamento de efluentes têxteis visando o seu reuso. 2011. 109f. Dissertação (Mestrado em Desenvolvimento e Meio Ambiente) - TROPEN, Universidade Federal do Piaui, 2011.

MORAIS, J. L. de. Estudo da potencialidade de processos oxidativos avançados, isolados e integrados com processos biológicos tradicionais, para tratamento de chorume de aterro sanitário. 2005. 207f. Tese (Doutorado em Química) - Universidade Federal do Paraná, 2005.

NOGUEIRA, R. F. P.; JARDIM, W. F. Heterogeneous photocatalysis and its environmental. Química Nova, v.21, n.1, p.69 72, 1998.

NOGUEIRA, R. F. P.; TROVÓ, A. G.; SILVA, M. R. A. Da; VILLA, R. D. fundamentos e aplicações ambientais dos processos fenton e foto-fenton. Química Nova, v.30, n.2, p.400-408, 2007.

NOGUEIRA, R. F. P.; GUIMARÃE, S. J. R. Photodegradation of dichloroacetic acid and 2,4-dichlorophenol by ferrioxalate $/ \mathrm{H}_{2} \mathrm{O}_{2}$ system. Water Research, v.34, p.895-901, 2000.

PARRA, S. Coupling of photocatalytic and biological process as a contribution to the detoxification ofwater: catalytic and technological aspects. Lusanne, 2001. Thesis (Docteur ès sciences techniques), École Polytechnique Féderale De Lausanne, EPFL, 2001.

PERALTA-ZAMORA, P.; ESPOSITO, E.; REYES, J.; DURÁN, N. Remediação de efluentes derivados da indústria de papel e celulose. Tratamento biológico e fotocatalítico. Química Nova, v.20(2), p.186-190, 1997.

PIGNATELLO, J. J. Dark and photoassisted Fe3+-catalyzed degradation of chlorophenoxy herbicides by hydrogen peroxide. Environ. Sci. Technol. v.26, p.944-951, 1992.

PREVOT, A. B.; PRAMAURO, E. Analytical monitoring of photocatalytic treatments, Degradation of 2,3,6-trichlorobenzoic acid in aqueous $\mathrm{TiO}_{2}$ dispersions. Talanta, v.48, p.847-857, 1999. 
PRUDEN, A. L.; OLLIS, D. F. Photoassisted heterogeneous catalysis $\square$ The degradation of trichloroethylene in water. Journal Catalysis, v.83, n.2, p.404 $\square$ 417, 1983a.

PRUDEN, A. L.; OLLIS, D. F. Degradation of chloroform by photoassisted heterogeneous catalysis in dilute aqueous suspensions of titanium dioxide. Environmental Science and Technology, v.17, n.10, p.628 $\square 631,1983 \mathrm{~b}$.

SAFARZADEH-AMIRI, A.; BOLTON, J. R.; CATER, S. R. Ferrioxalate-mediated photodegradation of organic pollutants in contaminated water. Wat. Res., v.31, p.787-798, 1997.

SARRIA, V.; PARRA, S.; ADLER, N.; PÉRINGER, P.; BENITEZ, N.; PULGARIN, C. Recent developments in the coupling of photoassisted and aerobic biological processes for the treatment of biorecalcitrant compounds. Catalysis Today. v.76, p.301-315, 2002.

SCHRANK, S. G.; JOSE, H. J.; MOREIRA, R. F. P. M.; SCHRODER, H. F. Applicability of Fenton and $\mathrm{H} 2 \mathrm{O} 2 / \mathrm{UV}$ reactions in the treatment of tannery wastewaters. Chemosphere, v.60, n.5, p.644 655, 2005.

SCOTT, J. P.; OLLIS, D. F. Integration of chemicaland biological oxidation processes for water treatment: review and recommendations. Environmental Progress. V.14, (2), p.88103, 1995.

SERPONE, N. Relative photonic efficiencies and quantum yields in heterogeneous photocatalysis. Photochem. Photobiol., v.104, p.1-12, 1997.

SILVA, L. P. Modificação e imobilização de TiO, visando a degradação de compostos orgânicos poluentes via o processo de fotocatálise heterogênea. 2007. 115f. Dissertação (Mestrado em Química) - Instituto de Química, USP, 2007.

SOUZA, S. J. O.; LOBO, T. M.; SABINO, A. L. O.; OLIVEIRA, S. B.; COSTA, O. S. Decomposição dos antirretrovirais lamivudina e zidovudina pelo processo fotofenton assistido no efluente de indústria farmoquímica. Revista Processos Químicos, v.4, n.7,

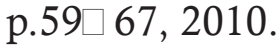

SOUZA, B. M. de. Avaliação de processos oxidativos avançados acoplados com carvão ativado granulado com biofilme para reuso de efluentes de refinaria de petróleo. 2010; 160f. Dissertação (Mestrado em Engenharia Química) - COPPE, Universidade Federal do Rio de Janeiro, 2010.

SPINKS, J. W. T.; WOODS, R. J. An Introduction to Radiation Chemistry, 3rded. John Wiley \& Sons: Nova York, 1990.

STOCKINGER, H.; HEINZLE, E.; KUT, O. M. Removal of chloro and nitro aromatic wastewater pollutants by ozonation and biotreatment. Environ. Sci. Technol. v.29, p.20162022, 1995.

TABRIZI, G. B.; MEHRVAR, M. Integration of advanced oxidation technologies and biological processes: recent developments, trends, and advances. Journal of Environmental Science And Health. Part A. v.39, (11-12), p.3029-3081, 2004.

TIBURTIUS, E. R. L.; PERALTA-ZAMORA, P. P. contaminação de águas por BTXS e processos utilizados na remediação de sítios contaminados. Quimica Nova, v.27, n.3, p.441-446, 2004. 
VANDEVIVERE, P. C.; BIANCHI, R.; VERSTRAETE, W. Treatment and reuse of wastewater from the testile wet $\square$ processing industry: review of emerging technologies. Journal of Chemical Technology and Biotechnology, v.72, n.4, p.289 $\square$ 302, 1998.

VIANNA, V. B.; TÔRRES, A. R.; AZEVEDO, E. B. Degradação de corantes ácidos por processos oxidativos avançados usando um reator com disco rotatório de baixa velocidade. Quimica Nova, v.31, n.6, p.1353-1358, 2008.

VINODGOPAL, K.; PELLER, J.; MAKOGON, O.; KAMAT, P.V., Ultrasonic mineralization of reactive textile azo dye, Remazol Black B. Water Research, v.32, p.3646-3650, 1998.

WANG, T. M.; WANG, H. Y.; XU, P.; ZHAO, X. C.; LIU, Y. L.; CHAO, S. The effect of properties of semiconductor oxide thin films on photocatalytic decomposition of dyeing waste water. Thin Solid Films, 334, p.103-108, 1998.

YEBER, M. C.; RODRIGUEZ, J.; FREER, J.; BAEZA, J.; DURÁN,N.; MANSILLA, H. D. Advanced oxidation of a pulp mill bleaching wastewater. Chemosphere. Oct;39(10), p.1679-88, 1999.

ZIOLLI, R. L.; JARDIM, W. F. Mechanism reactions of photodegradation of organic compounds catalyzed by $\mathrm{TiO}_{2}$. Química Nova. v.21, n.3, p.319 $\square 25,1998$.

ZUO, Y.; HOIGNÉ, J. Formation of hydrogen peroxide and depletion of oxalic acid in atmospheric water by photolysis of iron(III)-oxalato complexes. Environ. Sci. Technol. v.26, p.1014-1022. 1992. 